\title{
Caracterização sensorial de cafés solúveis comerciais por Perfil Flash ${ }^{1}$
}

\section{Sensory characterization of commercial soluble coffees by Flash Profile}

\author{
Marcela Lika Kobayashi ${ }^{2 *}$; Marta de Toledo Benassi ${ }^{3}$
}

\section{Resumo}

Dentro do competitivo mercado atual, há demanda por métodos mais simples na descrição de produtos. O Perfil Flash (Flash Profile) é uma combinação do levantamento de atributos do Perfil Livre com um método de ordenação, baseado na apresentação simultânea de todas as amostras a serem avaliadas, proporcionando uma descrição e discriminação rápida de um conjunto de produtos. Dessa forma, o presente estudo visou empregar o método de Perfil Flash para caracterizar sensorialmente cafés solúveis comerciais. Quatro cafés solúveis selecionados por apresentar diversidade de processo e composição foram avaliados por 32 julgadores, em uma única sessão. As bebidas foram preparadas com $28 \mathrm{~g}$ de café solúvel para $1000 \mathrm{~mL}$ de água filtrada, e adicionadas de 9,5\% de sacarose. Primeiramente, todos os cafés foram apresentados simultaneamente aos julgadores para o desenvolvimento da lista de atributos. Em discussão individual, os julgadores foram assistidos na definição dos atributos e na elaboração da ficha de avaliação. Posteriormente, as quatro bebidas foram apresentadas simultaneamente ao julgador, que as ordenou em ordem crescente quanto à intensidade de cada atributo da sua ficha de avaliação. Os resultados foram avaliados por Análise Procrustes Generalizada. Os atributos mais relevantes na descrição e discriminação das bebidas de cafés adoçadas foram cor marrom, aroma e sabor de café, gosto amargo, gosto doce e presença de oleosidade na superfície da bebida. O método Perfil Flash mostrou ser eficiente na descrição e discriminação de uma matriz alimentar complexa, cafés solúveis, apresentando consenso entre os julgadores, e rapidez na avaliação.

Palavras-chave: Análise procrustes generalizada, cor marrom, aroma e sabor de café, gosto amargo, gosto doce

\begin{abstract}
In today's competitive market, there is a lack of simple methods for sensory characterization of products. The Flash Profile is a combination of the Free Choice Profiling terms selection with a ranking method, based on the simultaneous presentation of all samples to be evaluated, providing a quick description and discrimination of a set of products. Thus, this study aimed to apply the Flash profile method on the characterization of commercial soluble coffees. Four soluble coffees selected by presenting diversity in the production process and composition were evaluated by 32 assessors in a single session. The coffee brews were prepared with $28 \mathrm{~g}$ of soluble coffee per $1000 \mathrm{~mL}$ of purified water, and added of $9.5 \%$ sucrose. Initially, the whole set of samples were presented simultaneously for the glossary development. In individual discussion, each assessor was assisted on the elaboration of individual score sheet with the definition of each attribute. Subsequently, the four coffee beverages were presented simultaneously to the assessor, who ordered the samples in ascending order for the intensity of each attribute on its score
\end{abstract}

1 Parte da Dissertação de Mestrado em Ciência e Tecnologia de Alimentos, Universidade Estadual de Londrina,UEL, Londrina, PR.

2 Discente de Mestrado Dept ${ }^{\circ}$ de Ciência e Tecnologia de Alimentos, UEL, Londrina, PR. E-mail: marcela.kobayashi@gmail.com

3 Prof $^{a} \operatorname{Dr}^{\mathrm{a}}$ do Dept $^{\circ}$ de Ciência e Tecnologia de Alimentos, UEL, Londrina, PR. E-mail: martatb@uel.br

Autor para correspondência 
sheet. The results were analyzed by Generalized Procrustes Analysis. The most relevant attributes in the description and discrimination of sweetened coffee brews were brown color, aroma and flavor of coffee, bitter taste, sweet taste and the presence of oil on the brew surface. The Flash Profile method was efficient on the description and discrimination of a complex food matrix as soluble coffee, presenting consensus among the assessors, and a fast assessment.

Key words: Generalized procrustes analysis, brown color, aroma and flavor of coffee, bitter taste, sweet taste

\section{Introdução}

Métodos de análise sensorial descritiva quantificam características de produtos quanto à aparência, sabor, aroma, textura e sensações residuais, usualmente empregando equipe selecionada e treinada para uso de escalas e avaliação dos atributos (DUTCOSKY, 2011; IAL, 2008). As etapas de busca por vocabulário consensual e treinamento são longas e cansativas, e podem necessitar de várias semanas a vários meses (SIEFFERMANN, 2000).

O Perfil Flash (Flash Profile), proposto por Sieffermann (2000), é um método descritivo que proporciona acesso mais rápido de classificação e descrição de um conjunto de produtos em relação ao método descritivo tradicional (DELARUE; SIEFFERMANN, 2004; TERHAAG, BENASSI, 2011). A técnica combina a etapa de levantamento de atributos individual do Perfil Livre (WILLIAMS; LANGRON, 1984), seguido da ordenação de cada atributo de um conjunto de produtos apresentados simultaneamente (LAWLESS; HEYMANN, 2010). Segundo Sieffermann (2000), a idéia da técnica é colocar o participante em frente à diversidade total do conjunto de produtos, solicitando ao julgador que se concentre na avaliação comparativa dos produtos. A rapidez do método é resultante de dois fatores: a avaliação comparativa simultânea das amostras e no fato de os julgadores utilizarem as próprias listas de atributos, eliminando a etapa de treinamento e procura por consenso no vocabulário (DELARUE; SIEFFERMANN, 2004; LAWLESS; HEYMANN, 2010; SIEFFERMANN， 2000). Alguns autores (DAIROU; SIEFFERMANN, 2002; DELARUE; SIEFFERMANN, 2004; RASON et al., 2006; SIEFFERMANN, 2000) descrevem ainda que os participantes devam ser de preferência julgadores treinados em análise descritiva ou experts da categoria do produto.

Como procedimento, Dairou e Sieffermann (2002) descrevem o emprego de uma sessão para desenvolvimento individual dos descritores e outra sessão, na qual os julgadores, informados de todos os descritores propostos pelos outros membros da equipe, poderiam alterar a sua lista individual antes da análise. Dessa forma, todos os membros da equipe tinham que concluir a primeira sessão para dar sequencia à etapa de ordenação. Considerando que o conceito do Perfil Livre é de que o julgador gera e utiliza os descritores que desejar, sem treinamento ou discussão com a equipe, Terhaag e Benassi (2011), em um estudo com bebidas de soja, sugeriram que o procedimento proposto para o Perfil Flash poderia ser individualizado e sintetizado em apenas uma sessão. Os autores utilizaram, ainda, julgadores sem experiência anterior em análise descritiva e obtiveram bom resultado.

Os dados do Perfil Flash só podem ser avaliados pela Análise Procrustes Generalizada (APG), tendo em vista que as matrizes de dados de cada provador têm tamanho diferenciado e que não há consenso nos atributos empregados, não sendo possível trabalhar com dados médios da equipe (OLIVEIRA; BENASSI, 2003). Assim o princípio da APG é aproximar as configurações de cada provador a uma configuração de consenso, de maneira a eliminar as variações no uso de escala, de diferentes intervalos de valores ou de interpretações diferenciadas dos atributos. A APG permite ainda detectar diferenças na percepção e falta de repetibilidade, e eliminar resultados de provadores que não apresentam consenso com a equipe (WILLIAMS; 
LANGRON, 1984). Na análise dos resultados, as dimensões do espaço de consenso são interpretadas pelas correlações dos atributos de cada provador (LAWLESS; HEYMANN, 2010).

Vignoli, Bassoli e Benassi (2011), estudando cafés solúveis produzidos a partir de cafés arábica e conilon com diferentes graus de torra e processos de extração, destacaram a variabilidade na composição devido a diferenças nas espécies e processos utilizados. No único estudo disponível sobre a composição de cafés solúveis comerciais brasileiros, Nogueira e Trugo (2003) verificaram grande variabilidade na composição dos produtos de diferentes marcas, que atribuíram tanto a diferenças na formulação dos blends quanto ao processamento.

$\mathrm{Na}$ literatura existem poucos estudos de análise sensorial com café solúvel (CALVIÑO; ZAMORA; SARCHI, 1996; GEEL; KINNEAR; KOCK, 2005; MORAES; BOLINI, 2010; NORONHA, 2003), e poucos trabalhos empregando Perfil Flash (BLANCHER et al., 2007; DAIROU; SIEFFERMANN, 2002, DELARUE; SIEFFERMANN, 2004; JAROS et al., 2009; LASSOUED et al., 2008; PERRIN; PAGÈS, 2009; POINOT et al., 2007; RASON et al., 2006; SIEFFERMANN, 2000; TAREA; CUVELIER; SIEFFERMANN, 2007; TERHAAG; BENASSI, 2011). Dessa forma, o presente estudo teve como objetivo a aplicação do Perfil Flash na caracterização sensorial de cafés solúveis comerciais brasileiros, avaliados como bebidas adoçadas.

\section{Material e Métodos}

\section{Caracterização das amostras}

Quatro produtos comerciais de café solúvel brasileiro de diferentes marcas, com diferenças na composição, processamento e nas características físico-químicas das bebidas, foram utilizados nesse estudo (Tabela 1).

Tabela 1. Caracterização físico-química das bebidas adoçadas de café solúvel.

\begin{tabular}{|c|c|c|c|c|c|c|c|}
\hline \multirow{2}{*}{\multicolumn{2}{|c|}{$\begin{array}{c}\text { Amostra / } \\
\text { Especificação dos fabricantes }\end{array}$}} & \multicolumn{5}{|c|}{ Características físico-químicas ${ }^{1,2}$} & \multirow[b]{2}{*}{$\mathrm{SST}^{5}$} \\
\hline & & $\mathrm{L}^{*}$ & $\mathrm{~h}^{\mathrm{o}}$ & $\mathrm{pH}$ & $\mathrm{ATT}^{3}$ & $\begin{array}{l}\text { Compostos } \\
\text { escuros }^{4} \\
\end{array}$ & \\
\hline A & $\begin{array}{l}\text { Café solúvel granulado, } \\
\text { proveniente de blend, } \\
\text { com denotação comercial } \\
\text { "forte" e "tradição". }\end{array}$ & $21,9 \pm 0,3^{\mathrm{b}}$ & $54,1 \pm 1,5^{\mathrm{c}}$ & $5,06 \pm 0,00^{\mathrm{a}}$ & $2,6 \pm 0,0^{c}$ & $0,434 \pm 0,002^{\mathrm{b}}$ & $12,3 \pm 0,0^{\mathrm{a}}$ \\
\hline B & $\begin{array}{l}\text { Café solúvel liofilizado, } \\
100 \% \text { arábica, com } \\
\text { categoria gourmet. }\end{array}$ & $22,0 \pm 0,1^{\mathrm{b}}$ & $62,6 \pm 1,1^{\mathrm{a}}$ & $5,03 \pm 0,01^{\mathrm{b}}$ & $3,0 \pm 0,1^{b}$ & $0,509 \pm 0,003^{\mathrm{a}}$ & $12,3 \pm 0,0^{\mathrm{a}}$ \\
\hline $\mathrm{C}$ & $\begin{array}{l}\text { Café solúvel em pó, } \\
\text { proveniente de blend, } \\
\text { com denotação comercial } \\
\text { "extra-forte" e "cremoso". }\end{array}$ & $21,3 \pm 0,1^{\mathrm{c}}$ & $57,5 \pm 1,3^{\mathrm{b}}$ & $4,99 \pm 0,00^{c}$ & $3,1 \pm 0,0^{b}$ & $0,430 \pm 0,002^{\mathrm{b}}$ & $12,3 \pm 0,0^{\mathrm{a}}$ \\
\hline $\mathrm{D}$ & $\begin{array}{l}\text { Café solúvel granulado, } \\
\text { proveniente de blend. }\end{array}$ & $23,4 \pm 0,1^{\mathrm{a}}$ & $62,8 \pm 0,3^{\mathrm{a}}$ & $4,86 \pm 0,01^{\mathrm{d}}$ & $3,4 \pm 0,1^{\mathrm{a}}$ & $0,375 \pm 0,001^{\mathrm{c}}$ & $12,3 \pm 0,0^{\mathrm{a}}$ \\
\hline
\end{tabular}

${ }^{1}$ Letras diferentes na mesma coluna indicam diferença significativa (Tukey, $\mathrm{p} \leq 0,05$ ).

${ }^{2}$ Valores médios (média \pm desvio-padrão) de três repetições.

${ }^{3}$ Acidez total titulável em $\mathrm{mL}$ de $\mathrm{NaOH} 0,1 \mathrm{~N} / 20 \mathrm{~mL}$ bebida.

${ }^{4}$ UA a $420 \mathrm{~nm}$.

${ }^{5}$ Sólidos solúveis totais em ${ }^{\circ}$ Brix.

Fonte: Elaboração dos autores. 
Considerando-se as concentrações de bebidas de café solúvel já descritas na literatura (MORAES; BOLINI, 2010; VIGNOLI; BASSOLI; BENASSI, 2011), determinou-se, em pré-testes, uma concentração adequada para a avaliação. As bebidas do estudo foram preparadas com $28 \mathrm{~g}$ de café solúvel para $1000 \mathrm{~mL}$ de água filtrada a $95^{\circ} \mathrm{C}$. As bebidas foram adicionadas de 9,5\% de sacarose, concentração descrita por Moraes e Bolini (2010) como conferindo a doçura ideal para café solúvel. Optou-se por servir as bebidas adoçadas uma vez que os participantes do estudo eram consumidores regulares de café, mas não eram julgadores treinados, e estavam habituados a consumir o produto com açúcar. As bebidas foram armazenadas em garrafas térmicas, servidas em, no máximo, duas horas após o preparo, mantendo sempre temperatura superior a $68^{\circ} \mathrm{C}$.

Para a caracterização das bebidas, a acidez titulável foi medida pelo método potenciométrico. $20 \mathrm{~mL}$ de bebida de café (a $25^{\circ} \mathrm{C}$ ), teve seu $\mathrm{pH}$ medido em um potenciômetro digital Hanna HI 2223 (Woonsocket, EUA), e foi titulado com solução de $\mathrm{NaOH} 0,1 \mathrm{~N}$ até $\mathrm{pH} 8,2$. Os resultados da acidez foram expressos em $\mathrm{mL}$ de $\mathrm{NaOH} 0,1 \mathrm{~N}$ gastos para $20 \mathrm{~mL}$ de bebida (BUENAVENTURASERRANO; CASTAÑO-CASTRILLÓN，2002; CECCHI, 2009). Os sólidos solúveis totais foram medidos em refratômetro digital Atago Pal-BX/ RI (Tokio, Japão), e expressos em ${ }^{\circ}$ Brix. A cor foi medida nos cafés acondicionados numa cubeta de polipropileno, utilizando iluminante D65, geometria 45/0 em colorímetro portátil Konica Minolta CR 400 (Tokio, Japão). Determinou-se também a absorvância das bebidas diluídas $(1 \mathrm{~mL}$ de bebida de café para $40 \mathrm{~mL}$ de água destilada) a $420 \mathrm{~nm}$ em espectrofotômetro UV-VIS Biochrom Libra S22 (Cambridge, Inglaterra), sugerida como estimativa de compostos escuros em bebidas de café por López-Galilea et al. (2008).
Caracterização dos julgadores e análise sensorial descritiva das bebidas

O estudo contou com a colaboração de uma equipe de 32 participantes (recrutados por questionário), a maioria do sexo feminino (78\%), jovens $(41 \%$ com idade inferior a 25 anos, e $56 \%$ entre 25 e 40 anos) e com alto grau de escolaridade (66\% com superior completo e $31 \%$ com $2^{\circ}$ grau completo). Dentre os participantes, $78 \%$ já haviam participado de testes sensoriais de aceitação (41\%), discriminativo (29\%) e descritivo (30\%). Todos os participantes eram consumidores regulares de café, $61 \%$ de torrado e moído e $39 \%$ de solúvel. Mais da metade dos participantes consumia pelo menos uma xícara de café/dia, 19\% consumiam entre três a cinco, e $28 \%$ consumiam café numa frequência superior ou inferior às propostas como resposta. Os participantes foram informados sobre os procedimentos do teste, conforme descrito no projeto cadastrado no Sistema Nacional de Ética em Pesquisa (Certificado de Apresentação para Apreciação Ética no 0167.0.268.000-10, aprovado no CEP/UEL no 193/2010), e assinaram um Termo de Consentimento Livre e Esclarecido.

Os testes foram realizados em cabines individuais em laboratório de análise sensorial. Aproximadamente $50 \mathrm{~mL}$ de cada bebida foram servidos aos julgadores em copos descartáveis de isopor codificados com número aleatório de três dígitos. Em todas as provas, as quatro amostras foram servidas simultaneamente, e a ordem de apresentação foi aleatorizada para cada julgador. Quando necessário, um novo conjunto de amostras foi apresentado a cada julgador, tanto na etapa de levantamento de atributos quanto na avaliação de ordenação, de maneira a fazer a prova sempre com o café quente.

No levantamento de atributos, os julgadores observaram as similaridades e diferenças em relação à aparência, aroma, sabor e textura do conjunto de bebidas de cafés solúveis, sendo instruídos a evitar termos hedônicos. Em conversa individual, 
cada julgador foi assistido no desenvolvimento do vocabulário, registrando os atributos encontrados e descrevendo-os sucintamente. Após o desenvolvimento da ficha de avaliação, as amostras foram novamente apresentadas e os julgadores ordenaram os produtos para cada termo descritivo em ordem crescente em relação à intensidade (TERHAAG; BENASSI, 2011).

Os dados do Perfil Flash foram analisados por Análise Procrustes Generalizada, utilizando-se o programa Senstools versão 2.3.28 (OP \& PRODUCT RESEARCH, 1998). Os dados foram inseridos na forma de 32 matrizes (uma por julgador) de quatro linhas (amostra) e o número de colunas variando de 5 a 11 (atributos). Como critério para avaliar a eficiência da equipe, foram consideradas as variâncias residuais de cada julgador, a configuração dos julgadores e as configurações das amostras por julgador. A análise final dos resultados descritivos foi baseada numa configuração de consenso das amostras, que foi interpretada analisando as correlações (de cada atributo com as dimensões 1, 2 e 3) para cada julgador. O critério utilizado na escolha dos atributos para análise, foi considerar os termos que apresentaram para cada julgador correlação, em módulo, igual ou superior a 0,60 , e que tivessem sido citados por um maior número de julgadores.

\section{Resultados e Discussão}

A Tabela 1 apresenta as características cor (luminosidade, $\mathrm{L}^{*}$, e tonalidade cromática, $\mathrm{h}^{\mathrm{o}}$ ), $\mathrm{pH}$, acidez total titulável (ATT), compostos escuros e sólidos solúveis totais (SST) das bebidas de café solúvel. Constam ainda algumas especificações dos fabricantes dos produtos descritas no rótulo, que indicavam diferenças nas matérias-primas (blend de arábica e conilon ou Gourmet, 100\% arábica) e processos de secagem (pó, granulado e liofilizado) utilizados.
Pela padronização no preparo, todas as bebidas apresentavam o mesmo teor de sólidos solúveis totais $\left(12,3^{\circ} \mathrm{Brix}\right)$. As bebidas diferiam quanto à cor ( $\mathrm{L}^{*}$ de 21,3 a 23,4 ; $\mathrm{h}^{\circ}$ de 54,1 a 62,8 ; compostos escuros de 0,375 a 0,509) e acidez ( $\mathrm{pH}$ de 2,6 a 3,4 , ATT de 2,6 a 3,4 mL de NaOH 0,1 N / 20mL bebida), indicando diferentes graus de torra (Tabela 1).

Numa avaliação da performance dos julgadores, o consenso pode ser confirmado pela baixa variância residual (inferior a $1 \%$ ) (Figura 1) para uma solução com 3 dimensões. Avaliando-se a configuração geral dos julgadores observou-se pouca dispersão, indicando comportamento pouco diferenciado entre os membros da equipe (Figura 2).

O comportamento foi similar ao relatado por Terhaag e Benassi (2011) na descrição de bebidas comerciais de soja por Perfil Flash, na qual se observou variância residual máxima de 0,6 \% por julgador para uma solução tridimensional. Em trabalhos de Perfil Livre, em geral, se descreve variância para os julgadores de 0,6\% até 3,5\% (BENASSI; DAMÁSIO, CECCHI, 1998; CALEGUER; MINIM; BENASSI, 2007; FERREIRA et al., 2009; KITZBERGER et al., 2011; OLIVEIRA; BENASSI, 2010; RICHTER et al., 2010). A menor variância no uso de Perfil Flash pode ser atribuída ao à facilidade do uso de escalas ordinais em comparação com o uso de escalas de intervalo. Richter et al. (2010) descreveram o mesmo comportamento comparando a performance de equipes de Análise Descritiva Quantitativa e Perfil Livre (variância residual máxima de 1,2 \% e $1,5 \%$, respectivamente) e com o desempenho da equipe de Análise Descritiva por Ordenação (ADO) (variância residual máxima de $0,5 \%$ ), que aplicava o procedimento de ordenação. 
Figura 1. Distribuição da variância residual (\%) dos julgadores na solução tridimensional. Julgadores: números de 1 a 32 .

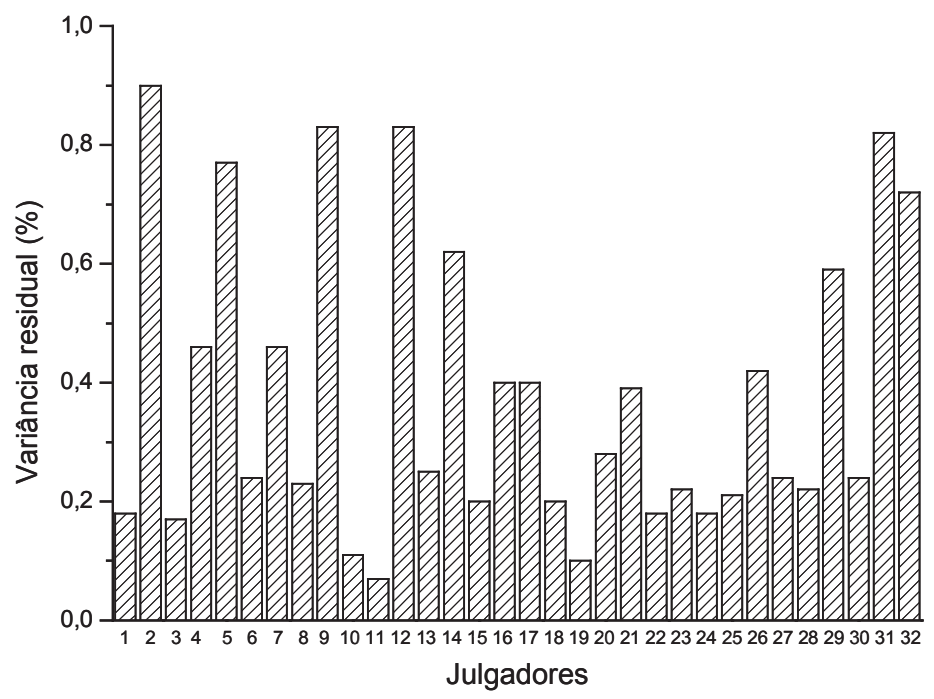

Fonte: Elaboração dos autores.

Figura 2. Configuração geral dos julgadores nas dimensões D1 e D2 (a) e D1 e D3 (b). Julgadores: números de 1 a 32.

(a)

(b)
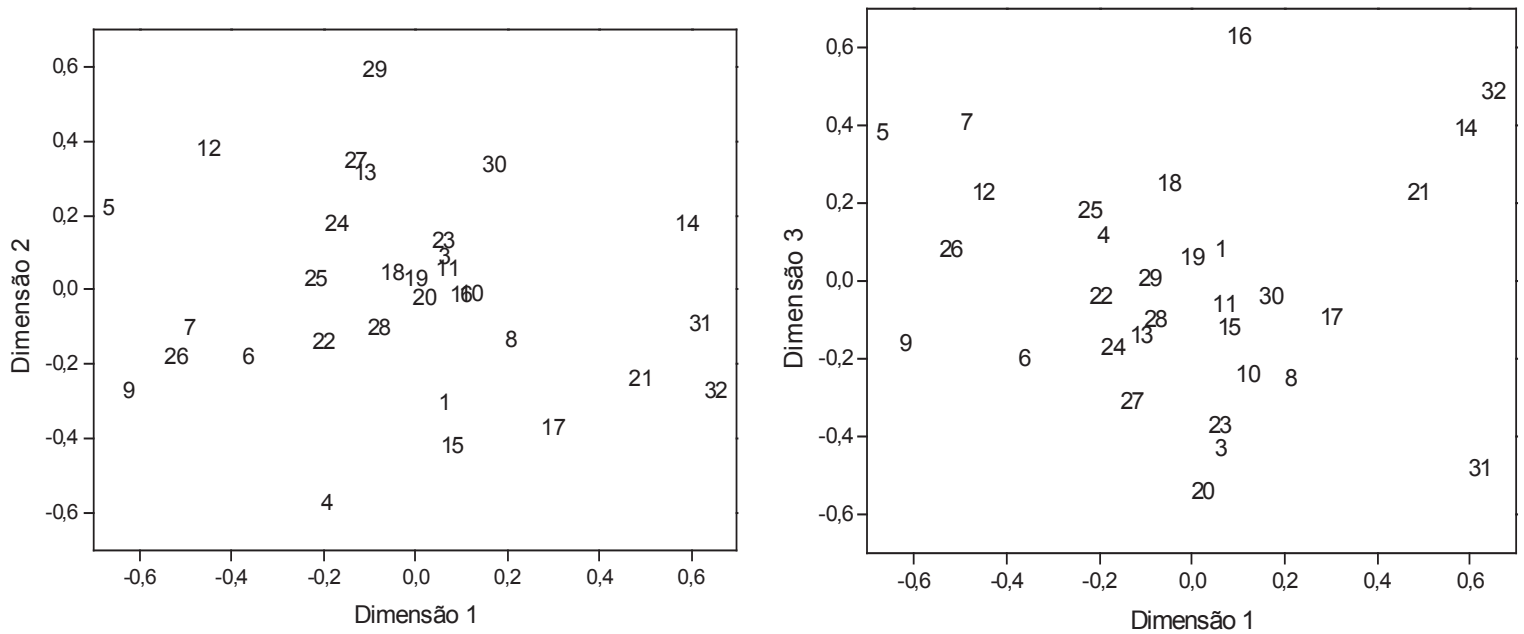

Fonte: Elaboração dos autores. 
Observou-se boa discriminação sensorial entre as amostras na solução com três dimensões $(87,8$ \% de explicação) (Tabela 2), e a configuração de consenso das amostras pode ser observada na Figura 3. Optou-se pela inclusão da terceira dimensão na análise, tendo em vista as altas correlações observadas para o atributo de oleosidade/ presença de partículas/ óleo na superfície da bebida para um número grande de julgadores (Tabela 3).

Tabela 2. Variância explicada (\%) da solução tridimensional para descrição de cafés solúveis.

\begin{tabular}{ccc}
\hline Dimensão & Consenso & Resíduo \\
\hline D1 & 50,9 & 5,5 \\
D2 & 19,7 & 4,1 \\
D3 & 17,2 & 2,6 \\
Total & 87,8 & 12,2 \\
\hline
\end{tabular}

Fonte: Elaboração dos autores.

Figura 3. Configuração de consenso das bebidas de café solúvel adoçadas (A, B, C e D) nas dimensões D1 e D2 (a), e D1 e D3 (b).

(a)

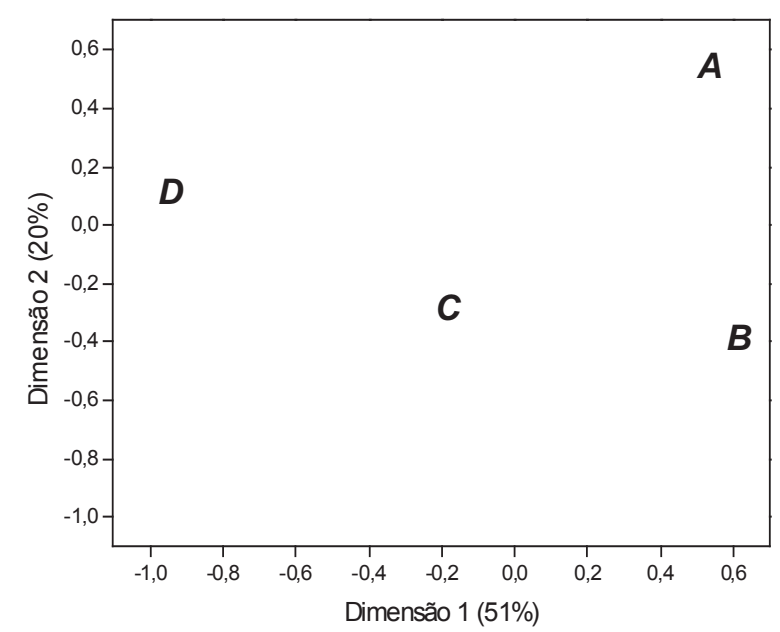

Fonte: Elaboração dos autores.

$\mathrm{Na}$ descrição de produtos por Perfil Flash, foi relatada explicação de $76 \%$ numa solução tridimensional para bebidas comerciais de soja (TERHAAG; BENASSI, 2011). Para soluções bidimensionais, foram descritas explicações de 69 \% a 89 \% na descrição de geléias e queijos quark, e na avaliação da textura de produtos assados de panificação (DAIROU; SIEFFERMANN, 2002, (b)

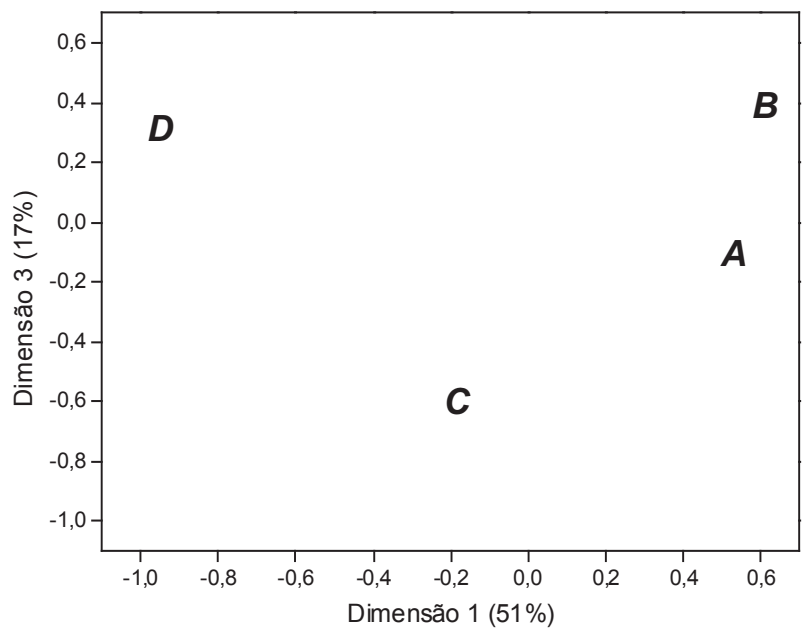

DELARUE; SIEFFERMANN, 2004; BLANCHER et al., 2007; LASSOUED et al., 2008).

Os termos descritivos mais relevantes na descrição das bebidas adoçadas estão apresentados na Tabela 3. Foram considerados de importância para descrição, os termos que apresentaram para cada julgador correlação, em módulo, igual ou superior a 0,60 , e que foram empregados de forma 
similar por um maior número de julgadores, com maior número de citações na Tabela 3. Diversos descritores atribuídos às bebidas em relação à cor, ao aroma e ao sabor (Tabela 3) foram coincidentes com os atributos de café solúvel encontrados na literatura para métodos descritivos convencionais (CALVIÑO. ZAMORA; SARCHI, 1996; MAMEDE et al., 2010).

Os atributos mais relevantes na descrição das bebidas de café solúvel adoçadas foram cor marrom, aroma e sabor de café, gosto amargo/ sabor de queimado (correlação positiva para dimensão 1); aroma doce/caramelo (correlação negativa para D1); gosto doce/sabor caramelo (correlação negativa para D1 e D2); presença de óleo/partículas/ oleosidade (correlação negativa para D3) (Figura 3 e Tabela 3).

Apesar de ter sido observada correlação acima de $|0,6|$ para atributos de textura (encorpado, denso, fluído, líquido) por muitos julgadores, não houve consenso no emprego dos mesmos. Esse comportamento pode ser atribuído a haver pouca diferença entre as amostras (que apresentavam o mesmo teor de sólidos solúveis totais) e/ou pela maior dificuldade na descrição e emprego desses atributos pelos julgadores (Tabelas 1 e 3).

A bebida $\mathrm{D}$, alocada à esquerda no gráfico de consenso, foi caracterizada por apresentar menor intensidade de cor marrom, aroma e sabor de café, gosto amargo/sabor de queimado (atributos com correlação negativa com D1) e maior intensidade de aroma doce/caramelo (atributos com correlação positiva com D1) (Figura 3 e Tabela 3). Estas características sensoriais são coincidentes com as características físico-químicas avaliadas, uma vez que essa bebida apresentou o menor valor de compostos escuros (0,375 UA), a maior luminosidade $(23,4)$ e maior acidez titulável $(3,4$ $\mathrm{mL}$ de $\mathrm{NaOH}$ 0,1 N / 20 mL) (Tabela 1). Pode-se associar essas características a um grau de torra menos intenso e/ou com maior presença de café conilon, que corresponderia a um café mais ácido e mais claro.

A bebida $\mathrm{C}$, caracterizada por possuir valores intermediários de luminosidade $(21,3)$, tonalidade cromática $(57,5)$, acidez titulável $(3,1 \mathrm{~mL}$ de $\mathrm{NaOH}$ $0,1 \mathrm{~N} / 20 \mathrm{~mL})$ e compostos escuros (0,403 UA) (Tabela 1), foi alocada mais ao centro do gráfico de consenso. A bebida também foi descrita como apresentando um perfil sensorial intermediário, com menor intensidade de cor marrom, aroma e sabor de café e gosto amargo/sabor de queimado que as bebidas A e B (discriminada pela D1), e maior presença de óleo na superfície (atributo com correlação negativa com D3) que observada para as bebidas A, B e D (Figura 3 e Tabela 3).

As bebidas $\mathrm{A}$ e $\mathrm{B}$, alocadas à direita do gráfico de consenso, foram descritas como possuindo cor marrom, gosto amargo/sabor de queimado, aroma e sabor de café mais intensos (atributos com correlação positiva com D1). Essas amostras apresentaram os menores valores para acidez titulável $(2,6 \mathrm{e}$ 3,0 mL de NaOH 0,1 N / 20mL, respectivamente) e altos valores para compostos escuros (Tabela 1), possivelmente, devido à maior presença de café arábica, e/ou um grau de torra mais intenso. Compostos com características ácidas, formados nas etapas iniciais da torra, são posteriormente degradados (DAGLIA et al., 2000; GINZ et al., 2000), ocorrendo também a carbonização de alguns componentes, o que acentua o sabor de queimado (MELO, 2004). No geral, as bebidas A e B foram descritas como similares, diferenciando-se pela menor intensidade de gosto doce e cor marrom (atributos com correlação negativa com D2) na amostra A, e maior presença de óleo/partículas (atributo com correlação negativa com D3) na amostra B (Figura 3 e Tabela 3). 
Tabela 3. Atributos melhor correlacionados $(|r| \geq 0,6)$ com as três primeiras dimensões (D1, D2 e D3) por julgador no Perfil Flash.

\begin{tabular}{|c|c|c|c|}
\hline $\mathrm{J}^{*}$ & Dimensão 1 & Dimensão 2 & Dimensão 3 \\
\hline 1 & $\begin{array}{l}\text { Cor marrom }(0,96) \text {; cor esverdeada }(-0,93) \text {; } \\
\text { transparência }(0,96) \text {; aroma torrado }(0,70) \text {; aroma } \\
\text { caramelo }(-0,93) \text {; sabor caramelo }(-0,70) \text {; sabor café } \\
\text { solúvel }(-0,70) \text {; sabor residual }(0,93) \text {; textura densa } \\
(-0,93)\end{array}$ & Gosto azedo $(0,92)$ & Presença de óleo $(-0,97)$ \\
\hline 2 & $\begin{array}{l}\text { Cor marrom }(0,96) \text {; aroma característico de café } \\
\text { solúvel }(0,96) \text {; sabor característico de café solúvel } \\
(0,96) \text {; cremosidade }(0,96)\end{array}$ & Gosto doce $(-0,72)$ & \\
\hline 3 & $\begin{array}{l}\text { Cor marrom }(0,93) \text {; transparência }(-0,93) \text {; aroma } \\
\text { de café }(0,96) \text {; gosto azedo }(-0,93) \text {; gosto amargo } \\
(0,96)\end{array}$ & Aroma de queimado $(-0,92)$ & $\begin{array}{l}\text { Presenca de óleo na superficie } \\
(-0,73)\end{array}$ \\
\hline 4 & $\begin{array}{l}\text { Cor marrom }(0,64) \text {; aroma caramelo }(-0,93) \text {; gosto } \\
\text { amargo }(0,93)\end{array}$ & & $\begin{array}{l}\text { Gordura na superfície }(-0,65) \text {; } \\
\text { aroma torrado }(-0,84)\end{array}$ \\
\hline 5 & Gosto amargo $(0,70)$ & $\begin{array}{l}\text { Brilhante }(-0,92) \text {; Cor marrom } \\
(-0,92) \text {; Adstringente }(-0,96) \text {; } \\
\text { Sabor residual amargo }(-0,96)\end{array}$ & $\begin{array}{l}\text { Película superficial }(-0,97) ; \\
\text { aroma de torrado }(-0,91)\end{array}$ \\
\hline 6 & $\begin{array}{l}\text { Opacidade }(0,93) \text {; aroma de café }(0,96) \text {; aroma de } \\
\text { queimado }(-0,93) \text {; gosto doce }(0,64) \text {; Corpo }(-0,64)\end{array}$ & $\begin{array}{l}\text { Cor marrom }(-0,92) \text {; sabor café } \\
(-0,64)\end{array}$ & $\begin{array}{l}\text { Óleo na superfície }(0,73) \text {; sabor } \\
\text { de queimado }(-0,91)\end{array}$ \\
\hline 7 & $\begin{array}{l}\text { Cor marrom }(0,64) \text {; aroma doce }(0,70) \text {; sabor } \\
\text { torrado }(0,70)\end{array}$ & $\begin{array}{l}\text { Oleosidade }(-0,92) ; \quad \text { gosto } \\
\text { doce }(-0,72) ; \text { sabor residual } \\
\text { queimado }(-0,64)\end{array}$ & $\begin{array}{l}\text { Aroma de grau de torra }(-0,97) \text {; } \\
\text { tempo de sabor residual } \\
\text { queimado }(-0,97)\end{array}$ \\
\hline
\end{tabular}

Cor marrom $(0,93)$; aroma torrado $(0,96)$; sabor

8 queimado $(-0,93)$; sabor característico de café solúvel $(-0,96)$; sabor aguado $(-0,70)$; espuma $(-0,64)$

\begin{tabular}{llll}
\hline 9 & $\begin{array}{l}\text { Opacidade }(0,64) \text {; gosto doce }(-0,64) \text {; sabor residual } \\
\text { torrado }(0,64)\end{array}$ & Cor marrom $(-0,92)$ & $\begin{array}{l}\text { Presença de partículas }(-0,97) ; \\
\text { aroma de torrado }(-0,91)\end{array}$ \\
\hline 10 & $\begin{array}{l}\text { Cor marrom }(0,93) ; \text { brilho }(0,93) ; \text { aroma doce } \\
(-0,96) ; \text { densidade }(0,64)\end{array}$ & Gosto azedo $(0,72)$ & Presença de partículas $(-0,71)$ \\
\hline
\end{tabular}

Cor marrom $(0,64)$; aroma caramelizado $(-0,69)$;

11 gosto amargo $(0,96)$; adocicado $(-0,96)$; corpo Aroma de queimado $(-0,64)$ $(-0,93)$

12 Aroma de pinhão cozido $(-0,69)$

Cor preta $(-0,92)$; aroma de café verde $(0,92)$; sabor residual adocicado $(-0,92)$; concentração $(-0,64)$

\begin{tabular}{llll}
\hline 13 & $\begin{array}{l}\text { Cor marrom }(-0,70) \text {; transparente }(0.96) ; \text { aroma } \\
\text { adocicado }(-0,96) \text {; doçura }(-0,96) ; \text { fluido }(0,64)\end{array}$ & $\begin{array}{l}\text { Presença de óleo na superfície } \\
(0,96)\end{array}$ & Sabor residual amargo $(-0,73)$ \\
\hline 14 & $\begin{array}{l}\text { Cor marrom }(0,70) \text {; aroma de café }(0,96) \text {; aroma de } \\
\text { queimado }(-0,96) \text {; sabor de café }(0,96) ; \text { encorpado } \\
(0,96)\end{array}$ & $\begin{array}{l}\text { Sabor residual de queimado } \\
(-0,72)\end{array}$ & \\
\hline
\end{tabular}

Cor marrom $(0,64)$; Partícula oleosa na superfície

$15 \quad(0,93)$; aroma de torrado $(0,70)$; aroma ácido $(-0,70)$; sabor torrado $(0,93)$; sabor residual ácido $(-0,93)$; líquido $(-0,66)$

Tonalidade marrom $(-0,70)$; tonalidade preta

$16(0,70)$; aroma de café torrado e moído $(0,69)$; aroma característico de café solúvel $(-0,69)$; gosto ácido $(-0,96)$; sabor de café torrado e moído $(0,96)$
Presença de gotículas de óleo $(-0,65)$; presença de corpo $(-0,97)$ 
continuação

Cor marrom $(0,93)$; aroma de café $(0,66)$; aroma

17 de queimado $(-0,66)$; sabor de café solúvel $(-0,93)$; sabor de café $(0,96)$; sabor de queimado $(-0,66)$; encorpado $(0,93)$

18 Aroma de torrado $(0,96)$; aroma doce $(-0,96)$; sabor de torrado $(0,96)$

Presença de espuma $(-0,66)$; resíduos sobrenadantes

$19(0,64)$; cor preta $(0,96)$; aroma de café $(0,96)$; sabor de caramelo $(-0,96)$

20 Cor marrom $(-0,64)$; cor preta $(0,64)$; aroma azedo $(-0,93)$; sabor de café fresco $(0,96)$; denso $(-0,66)$

Cor preta $(0,93)$; aroma de erva verde $(-0,96)$;

21 aroma de café $(0,96)$; sabor de café $(0,66)$; sabor de erva verde $(-0,96)$; fluido $(-0,70)$

Presença de espuma $(-0,66)$; gosto amargo $(0,93)$;

22 gosto ácido $(-0,93)$; sabor frutado $(-0,93)$; fluido $(-0,69)$

Presença de espuma $(0,93)$; presença de camada esbranquiçada $(-0,64)$; aroma de café $(0,69)$; sabor residual doce $(0,96)$; sabor adocicado $(0,93)$; encorpado $(0,66)$

24 Aroma doce $(0,66)$; gosto doce $(0,96)$; gosto amargo $(-0,64)$; ralo $(0,96)$

25 Aparência encorpada $(0,96)$; aroma ácido $(-0,96)$; gosto ácido $(-0,70)$; adstringente $(0,64)$

Cor preta $(0,64)$; Aroma chocolate ao leite $(0,64)$;

26 aroma de baunilha $(0,64)$; sabor de remédio $(0,70)$; sabor mel $(-0,96)$

27 Aroma de biju $(-0,96)$; sabor de pinhão $(-0,96)$; sabor de café $(0,96)$; denso $(-0,64)$

28 Cor marrom $(0,64)$; gosto amargo $(0,66)$; aguado $(-0,93)$

29

Opacidade $(-0,64)$; aroma de café solúvel $(-0,69)$; aroma de queimado $(-0,66)$;

Cor marrom $(0,64)$; aroma de café solúvel $(0,69)$;

30 aroma de impurezas $(-0,69)$; sabor de café solúvel $(0,69)$; sabor de estragado $(-0,96)$; viscoso $(-0,93)$
Presença de camada oleosa $(-0,97)$

Cor caramelo (-0,64); sabor Translucidez $(0,73)$; Presença residual $(0,72) \quad$ de óleo $(-0,97)$

Opaca $(0,92) ;$ aroma de Gosto amargo $(-0,91)$

queimado $(0,72)$
Cor marrom (-0,64); Aroma de

grau de torra $(-0,64)$; gosto doce Oleosidade $(-0,73)$

$(0,92)$

Cor marrom $(-0,92)$

Cor preta $(-0,92)$; aroma de queimado $(0,92)$

Cor marrom $(-0,64)$; gosto doce $(-0,92)$

Presença de óleo queimado

$(-0,64)$; aroma de mel $(0,64)$; Líquida $(-0,97)$

sabor baunilha $(-0,92)$

Cor marrom $(-0,92)$

Sabor residual azedo $(-0,73)$

Aroma de mel $(0,72)$; Sabor de

chá $(-0,64)$

Cor marrom $(0,96)$; cor preta(-0,96); sabor adocicado $(-0,72)$; sabor queimado $(0,72)$; adstringente $\quad(0,72)$; acidez $(0,72)$; encorpado $(-0,96)$

Oleosidade $(-0,65)$

Sabor de açúcar mascavo Aspecto gorduroso $(-0,71)$

Cor marrom $(0,93)$; aroma de café $(0,93)$; aroma

31 de caramelo $(-0,96)$; sabor de mel $(-0,93)$; sabor de queimado $(0,96)$; adstringente $(-0,93)$; encorpado $(0,93)$

Cor preta $(0,96)$; aroma de café $(0,96)$; aroma de

32 caramelo $(-0,96)$; aroma de queimado $(-0,66)$; sabor adocicado $(-0,96)$; sabor de café $(0,96)$

*Os julgadores foram indicados por números de 1 a 32 .

Fonte: Elaboração dos autores. 


\section{Conclusões}

O método descritivo Perfil Flash, aplicado em uma única sessão com julgadores não-treinados, mostrou ser eficiente na rápida caracterização sensorial de um conjunto de bebidas adoçadas de cafés solúveis comerciais.

\section{Agradecimentos}

À CAPES e ao CNPq pelas bolsas de Mestrado e Produtividade em Pesquisa, e à equipe de julgadores.

\section{Referências}

BENASSI, M. T.; DAMÁSIO, M. H.; CECCHI, M. Avaliação sensorial de vinhos Riesling Itálico nacionais utilizando Perfil Livre. Ciência e Tecnologia de Alimentos, Campinas, v. 18, n. 3, p. 265-270, 1998.

BLANCHER, G.; CHOLLET, S.; KESTELOOT, R.; NGUYEN HOANG, D.; CUVELIER, G.; SIEFFERMANN, J. M. French and Vietnamese: How do they describe texture characteristics of the same food? A case study with jellies. Food Quality and Preference, Barking, v. 18, n. 3, p. 560-575, 2007.

BUENAVENTURA-SERRANO, C. E.; CASTAÑOCASTRILLÓN, J. J. Influencia de la altitud en la calidad de la bebida de muestras de café procedente del ecotopo 206B en Colombia. Cenicafé, Chinchiná, v. 53, n. 2, p. 119-131, 2002.

CALEGUER, V. F.; MINIM, V. P. R.; BENASSI, M. T. Impacto da embalagem do preparado sólido para refresco sabor laranja na intenção de compra do consumidor. Brazilian Journal of Food Technology, Campinas, v. 10, n. 3, p. 159-168, 2007.

CALVIÑO, A. M.; ZAMORA, M. C.; SARCHI, M. I. Principal components and cluster analysis for descriptive sensory assessment of instant coffee. Journal of Sensory Studies, Trumbull, v. 11, n. 3, p. 191-210, 1996.

CECCHI, H. M. Fundamentos teóricos e práticos em análise de alimentos. 2. ed. rev. Editora Unicamp. Campinas: Unicamp, 2009.

DAGLIA, M.; PAPETTI, A.; GREGOTTI, C.; BERTÉ, F.; GAZZANI, G. In vitro antioxidant and ex vivo protective activities of green and roasted coffee. Journal of Agricultural and Food Chemistry, Washington, v. 48, n. 5, p. 1449-1454, 2000.
DAIROU, V.; SIEFFERMANN, J. M. A comparison of 14 jams characterized by conventional profile and a quick original method, the flash profile. Journal of Food Science, Chicago, v. 67, n. 2, p. 826-834, 2002.

DELARUE, J.; SIEFFERMANN, J. M. Sensory mapping using flash profile. Comparison with a conventional descriptive method for the evaluation of the flavour of fruit dairy products. Food Quality and Preference, Barking, v. 15, n. 4, p. 383-392, 2004.

DUTCOSKY, S. D. Análise sensorial de alimentos. 2. ed. Curitiba: Champagnat, 2011. 426 p.

FERREIRA, E. L.; LENCIONI, C.; BENASSI, M. T.; BARTH, M. O.; BASTOS, D. H. M. Descriptive sensory analysis and acceptance of stingless bee honey. Food Science and Technology International, London, v. 15, n. 3, p. 251-258, 2009.

GEEL, L.; KINNEAR, M.; KOCK, H. L. Relating consumer preferences to sensory attributes of instant coffee. Food Quality and Preference, Barking, v. 16, n. 4, p. 237-244, 2005.

GINZ, M.; BALZER, H. H.; BRADBURY, A. G. W.; MAIER, H. G. Formation of aliphatic acids by carbohydrate degradation during roasting of coffee. European Food Research and Technology, Berlin, v. 211, n. 6, p. 404-410, 2000.

INSTITUTO ADOLFO LUTZ - IAL. Normas analiticas do Instituto Adolfo Lutz: métodos físico-químicos para análise de alimentos. São Paulo: IAL, 2008, 1020 p.

JAROS, D.; THAMKE, I.; RADDATZ, H.; ROHM, H. Single cultivar cloudy juice from table apples: An attempt to identify the driving force for sensory preference. European Food Research and Technology, Berlin, v. 229, n. 1, p. 51-61, 2009.

KITZBERGER, C. S. G.; SCHOLZ, M. B. S.; SILVA, J. B. G. D.; BENASSI, M. T. Caracterização sensorial de cafés arábica de diferentes cultivares produzidos nas mesmas condições edafoclimáticas. Brazilian Journal of Food Technology, Campinas, v. 14, p. 39-48, 2011.

LASSOUED, N.; DELARUE, J.; LAUNAY, B.; MICHON, C. Baked product texture: correlations between instrumental and sensory characterization using flash profile. Journal of Cereal Science, London, v. 48, n. 1, p. 133-143, 2008.

LAWLESS, H. T.; HEYMANN, H. Sensory evaluation of food: principles and practices. New York: Springer, $2010.850 \mathrm{p}$. 
LÓPEZ-GALILEA， I.; DE PEÑA, M.; CID, C. Application of multivariate analysis to investigate potential antioxidants in conventional and torrefacto roasted coffee. European Food Research and Technology, v. 227, n. 1, p. 141-149, 2008.

MAMEDE, M. E. O.; PERAZZO, K. K.; MACIEL, L. F.; CARVALHO, L. D. Avaliação sensorial e química de café solúvel descafeinado. Alimentos e Nutrição, Araraquara, v. 21, n. 2, p. 311-324, 2010.

MELO, W. L. B. A importância da informação sobre o grau de torra do café e sua influência nas características organolépticas da bebida. São Carlos: EMBRAPA, 2004. (Comunicado técnico, 58). Disponível em: <http://www. infoteca.cnptia.embrapa.br/handle/doc/30170>. Acesso em: 04 jul. 2012.

MORAES, P. C. B. T.; BOLINI, H. M. A. Different sweeteners in beverages prepared with instant and roasted ground coffee: Ideal and equivalent sweetness. Journal of Sensory Studies, Trumbull, v. 25, n. 1, p. $215-$ 225,2010

NOGUEIRA, M.; TRUGO, L. C. Distribuição de isômeros de ácidos clorogênicos e teores de cafeína e trigonelina em cafés solúveis brasileiros. Ciência $e$ Tecnologia de Alimentos, Campinas, v. 23, n. 2, p. 296299, 2003.

NORONHA, R. L. F. A expectativa do consumidor e sua influência na aceitação e percepção sensorial de café solúvel. 2003. Tese (Doutorado em Tecnologia de Alimentos) - Universidade Estadual de Campinas, Campinas.

OLIVEIRA, A. P. V.; BENASSI, M. T. Avaliação sensorial de pudins de chocolate com açúcar e dietéticos por Perfil Livre. Ciência e Agrotecnologia, Lavras, v. 34, n. 1, p. 146-154, 2010.

Perfil Livre: uma opção para análise sensorial descritiva. Boletim da Sociedade Brasileira de Ciência e Tecnologia de Alimentos, Campinas, v. 37, n. 1, p. 66-72, 2003.

OP \& PRODUCT RESEARCH, Senstools Versão 2.3. Utrecht: OP \& P Product Research, 1995-1998. Conjunto de Programas. 1CD-ROM.
PERRIN, L.; PAGÉS, J. Construction of a product space from the ultra-flash profiling method: application to 10 red wines from the Loire valley. Journal of Sensory Studies, Trumbull, v. 24, n. 3, p. 372-395, 2009.

POINOT, P.; GRUA-PRIOL， J.; ARVISENET, G.; RANNOU, C.; SEMENOU, M.; LE BAIL, A.; PROST, C. Optimisation of HS-SPME to study representativeness of partially baked bread odorant extracts. Food Research International, Essex, v. 40, n. 9, p. 1170-1184, 2007.

RASON, J.; LÉGER, L.; DUFOUR, E.; LEBECQUE, A. Relations between the know-how of small-scale facilities and the sensory diversity of traditional dry sausages from the Massif Central in France. European Food Research and Technology, Berlin, v. 222, n. 5-6, p. 580-589, 2006.

RICHTER, V. B.; ALMEIDA, T. C. A.; PRUDENCIO, S. H.; BENASSI, M. T. Proposing a ranking descriptive sensory method. Food Quality and Preference, Barking, v.21, n. 6, p. 611-620, 2010.

SIEFFERMANN, J. M. Le profil flash: un outil rapide et innovant d'évaluation sensorielle descriptive. In: AGORAL, 12., 2000, Montpellier. Anais... Paris: Lavoisier, 2000. p. 335-340.

TAREA, S.; CUVELIER, G.; SIEFFERMANN, J. M. Sensory evaluation of the texture of 49 commercial apple and pear purees. Journal of Food Quality, Ames, v. 30, n. 6, p. 1121-1131, 2007.

TERHAAG, M. M.; BENASSI, M. T. Perfil flash: uma opção para análise descritiva rápida. Brazilian Journal of Food Technology, Campinas, n. 14, p. 140-151, 2011. Edição Especial.

VIGNOLI, J. A.; BASSOLI, D. G.; BENASSI, M. T. Antioxidant activity, polyphenols, caffeine and melanoidins in soluble coffee: the influence of processing conditions and raw material. Food Chemistry, Barking, v. 124, n. 3, p. 863-868, 2010.

WILLIAMS, A. A.; LANGRON, S. P. The use of the free-choice profiling for the evaluation of commercials ports. Journal of the Science of Food and Agriculture, Oxford, v. 35, n. 5, p. 558-568, 1984. 